\title{
Numerical study of dynamic Smagorinsky models in large-eddy simulation of the atmospheric boundary layer: Validation in stable and unstable conditions
}

\author{
Jan Kleissl, ${ }^{1,2}$ Vijayant Kumar, ${ }^{1}$ Charles Meneveau, ${ }^{3}$ and Marc B. Parlange ${ }^{1,4}$ \\ Received 23 October 2005; revised 12 April 2006; accepted 2 May 2006; published 24 June 2006.
}

[1] Large-eddy simulation (LES) of atmospheric boundary layer (ABL) flow is performed over a homogeneous surface with different heat flux forcings. The goal is to test the performance of dynamic subgrid-scale models in a numerical framework and to compare the results with those obtained in a recent field experimental study (HATS (Kleissl et al., 2004)). In the dynamic model the Smagorinsky coefficient $c_{s}$ is obtained from test filtering and analysis of the resolved large scales during the simulation. In the scale-invariant dynamic model the coefficient is independent of filter scale, and the scaledependent model does not require this assumption. Both approaches provide realistic results of mean vertical profiles in an unstable boundary layer. The advantages of the scale-dependent model become evident in the simulation of a stable boundary layer and in the velocity and temperature spectra of both stable and unstable cases. To compare numerical results with HATS data, a simulation of the evolution of the ABL during a diurnal cycle is performed. The numerical prediction of $c_{s}$ from the scale-invariant model is too small, whereas the coefficients obtained from the scale-dependent version of the model are consistent with results from HATS. LES of the ABL using the scale-dependent dynamic model give reliable results for mean profiles and spectra at stable, neutral, and unstable atmospheric stabilities. However, simulations under strongly stable conditions (horizontal filter size divided by Obukhov length $>3.8$ ) display instabilities due to basic flaws in the eddy viscosity closure, no matter how accurately the coefficient is determined.

Citation: Kleissl, J., V. Kumar, C. Meneveau, and M. B. Parlange (2006), Numerical study of dynamic Smagorinsky models in largeeddy simulation of the atmospheric boundary layer: Validation in stable and unstable conditions, Water Resour. Res., 42, W06D10W06D10, doi:10.1029/2005WR004685.

\section{Introduction}

[2] In large-eddy simulation (LES) of turbulent flows, a subgrid-scale (SGS) model accounts for the effect of the small scales (smaller than the grid size $\Delta$ ) on the (simulated) resolved scales. Resolved scales are defined conceptually by filtering the velocity and scalar fields at the grid scale

$$
\widetilde{\mathbf{u}}(\mathbf{x})=\int \mathbf{u}\left(\mathbf{x}^{\prime}\right) F_{\Delta}\left(\mathbf{x}-\mathbf{x}^{\prime}\right) d \mathbf{x}^{\prime}
$$

where $\widetilde{\mathbf{u}}$ is the filtered velocity and $F_{\Delta}$ is the (homogeneous) filter function at scale $\Delta$. The most commonly used

\footnotetext{
${ }^{1}$ Department of Geography and Environmental Engineering and Center for Environmental and Applied Fluid Mechanics, Johns Hopkins University, Baltimore, Maryland, USA.

${ }^{2}$ Now at Department of Earth and Environmental Sciences, New Mexico Institute of Mining and Technology, Socorro, New Mexico, USA.

${ }^{3}$ Department of Mechanical Engineering and Center for Environmental and Applied Fluid Mechanics, John Hopkins University, Baltimore, Maryland, USA.

${ }^{4}$ School of Architecture, Civil and Environmental Engineering, Ecole Polytechnique Fédérale de Lausanne, Lausanne, Switzerland.

Copyright 2006 by the American Geophysical Union. 0043-1397/06/2005WR004685\$09.00
}

approach for parameterization of the SGS stress $\tau_{i j}=$ $\widetilde{u_{i} u_{j}}-\widetilde{u}_{i} \widetilde{u}_{j}$ is the Smagorinsky model [Smagorinsky, 1963]:

$$
\tau_{i j}^{\mathrm{Smag}}-\frac{1}{3} \tau_{k k} \delta_{i j}=-2 \nu_{T} \widetilde{S}_{i j}, \quad \nu_{T}=\left(c_{s}^{(\Delta)} \Delta\right)^{2}|\widetilde{S}|
$$

$\widetilde{S}_{i j}$ is the strain rate tensor, $|\widetilde{S}|=\sqrt{2 \widetilde{S}_{i j} \widetilde{S}_{i j}}$ is its magnitude, and $\nu_{T}$ is the eddy viscosity. The Smagorinsky model includes a parameter $c_{S}^{(\Delta)}$, the Smagorinsky coefficient, which needs to be specified to complete the closure. Accurate specification of this parameter is of paramount importance, since it determines the magnitude of the mean rate of SGS dissipation of kinetic energy, $\Pi_{\Delta}=-\left\langle\tau_{i j} \widetilde{S}_{i j}\right\rangle$. In traditional LES of atmospheric boundary layers, $c_{s}^{(\Delta)}$ is deduced from phenomenological theories of turbulence [Lilly, 1967; Mason, 1994] and also from models for the effects of stratification and shear upon the turbulence [Hunt et al., 1988; Deardorff, 1980; Canuto and Cheng, 1997; Redelsperger et al., 2001]. As a consequence, in simulations $c_{S}^{(\Delta)}$ is based on predetermined expressions that relate $c_{s}^{(\Delta)}$ to flow parameters such as the Kolmogorov constant $c_{k}$, the ratio of filter scale to distance to the ground and/or to the Obukhov length, etc.

[3] In an important development in turbulence theory and modeling, Germano et al. [1991] proposed a model entail- 
ing dynamic determination of $c_{s}^{(\Delta)}$. In the "dynamic model," selected features of the numerically computed large-scale fields are analyzed during the simulation to deduce the unknown model coefficient, instead of obtaining it from predetermined expressions. The rationale for the dynamic model is that the resolved scales in a simulation may reflect the effects of phenomena such as stratification, coherent structures, or wall blocking and their complex interactions more realistically than available turbulence theories.

[4] The dynamic model is based on the Germano identity [Germano, 1992],

$$
L_{i j} \equiv \overline{\widetilde{u}_{i} \widetilde{u}_{j}}-\overline{\widetilde{u}}_{i} \overline{\widetilde{u}}_{j}=T_{i j}-\overline{\tau_{i j}}
$$

where $L_{i j}$ is the resolved stress tensor and $T_{i j}=\overline{\overline{u_{i} u_{j}}}-\overline{\widetilde{u}_{i}} \overline{\widetilde{u}}_{j}$ is the subgrid stress at a test filter scale $\alpha \Delta$ (an overline denotes test filtering at a scale $\alpha \Delta$ ). In simulations, $\alpha$ is typically chosen to be equal to 2 . Applying this procedure and replacing $T_{i j}$ and $\tau_{i j}$ by their respective prediction from the Smagorinsky model, one obtains:

$$
L_{i j}-\frac{1}{3} \delta_{i j} L_{k k}=\left(c_{s}^{(\Delta)}\right)^{2} M_{i j}
$$

where

$$
M_{i j}=2 \Delta^{2}\left(\overline{|\widetilde{S}| \widetilde{S}_{i j}}-\alpha^{2} \beta|\overline{\widetilde{S}}| \widetilde{\widetilde{S}}_{i j}\right)
$$

and

$$
\beta=\frac{\left(c_{s}^{(\alpha \Delta)}\right)^{2}}{\left(c_{s}^{(\Delta)}\right)^{2}}
$$

is the ratio of coefficients at test and grid filter scales. Assuming scale invariance of the coefficient, namely

$$
\beta=1, \quad \text { or } \quad c_{s}^{(\Delta)}=c_{s}^{(\alpha \Delta)},
$$

Equation (4) can be solved for $c_{s}^{(\Delta)}$ by minimizing the square error averaged over all independent tensor components [Lilly, 1992]

$$
\left(c_{s}^{(\Delta)}\right)^{2}=\frac{\left\langle L_{i j} M_{i j}\right\rangle}{\left\langle M_{i j} M_{i j}\right\rangle} .
$$

Angle bracktes denote averaging in some spatial [Ghosal et al., 1995] or temporal domain [Meneveau et al., 1996]. For further details about the dynamic model, see Meneveau and Katz [2000], Piomelli [1999], and Kleissl et al. [2004, hereinafter referred to as KPM04].

[5] While the dynamic model provides realistic predictions of $c_{s}^{(\Delta)}$ when the flow field is sufficiently resolved (that is, the filter scale is much smaller than the turbulence integral scale), it was found in a posteriori [Porté-Agel et al., 2000, hereinafter referred to as POR] and a priori tests (KPM04) that $c_{s}^{(\Delta)}$ is underpredicted both near the wall and in stably stratified flows. POR attributed this weakness to the assumption of scale invariance (equation (7)) and proposed a dynamic model in which the coefficient is scale-dependent. In this modification of the dynamic model a second filter is applied at scale $\alpha^{2} \Delta$ (denoted by a hat) in addition to the filter at $\alpha \Delta$ producing an equation analogous to equation (4):

$$
\begin{aligned}
Q_{i j}-\frac{1}{3} \delta_{i j} Q_{k k} & =\left(c_{s}^{(\Delta)}\right)^{2} N_{i j}, \text { where } Q_{i j}=\widehat{\widetilde{u}_{i} \widetilde{u}_{j}}-\widehat{\widetilde{u}_{i}} \widehat{\widetilde{u}_{j}} \\
N_{i j} & =2 \Delta^{2}\left(|\widehat{S}| \widetilde{S}_{i j}-\alpha^{4} \beta^{2}|\widehat{S}| \widehat{\widetilde{S}}_{i j}\right) .
\end{aligned}
$$

It has been assumed here that $\beta$ is the same in the intervals between grid and test filter, and between test and second test filter scales, that is

$$
\frac{c_{s}^{\left(\alpha^{2} \Delta\right)}}{c_{s}^{(\alpha \Delta)}}=\frac{c_{s}^{(\alpha \Delta)}}{c_{s}^{(\Delta)}},
$$

which implies that

$$
\frac{c_{s}^{\left(\alpha^{2} \Delta\right)}}{c_{s}^{(\Delta)}}=\beta^{2}
$$

(see POR for more details). At this stage the two equations (4) and (9) can be solved for the two unknowns $c_{s}^{(\Delta)}$ and $\beta$. For further details on the scale-dependent dynamic model, see POR and KPM04.

[6] The scale-dependent dynamic model was applied, together with planar averaging, to LES of neutral atmospheric boundary layer flow (see POR), demonstrating an improved prediction of $c_{s}^{(\Delta)}$. As a consequence, more realistic results for mean velocity gradients and streamwise energy spectra were obtained. Also, in a priori tests (KPM04) of field experimental data (Horizontal Array Turbulence Study (HATS) [Horst et al., 2003]), the scaledependent model gave much improved predictions of $c_{s}^{(\Delta)}$ not only in neutral but also under unstable and stable atmospheric stability.

[7] It is important to note that even a perfect prediction of $c_{S}$ cannot simultaneously produce the correct SGS dissipation, SGS stress, and SGS force [Pope, 2000; Meneveau, 1994] and that the correlation between SGS stress tensor and filtered strain rate tensor is weak leading to poor performance of the Smagorinsky model in a priori testing [McMillan and Ferziger, 1979; Liu et al., 1994; Bastiaans et al., 1998; Higgins et al., 2003]. Indeed both dynamic SGS models examined in the paper cannot improve the stress-strain correlations, since the models considered only affect the constant $c_{s}$. In Figure 8 of Kleissl et al. [2003] we showed explicitly that the mean SGS fluxes would not be predicted accurately when the mean dissipation is predicted correctly. Despite these limitations, the widespread use of the eddy viscosity closure in the simulation of atmospheric flows justifies further research on the Smagorinsky model.

[8] In the present study, numerical predictions for $c_{s}^{(\Delta)}$ will be compared to measurements from HATS, and the effects of the SGS model on the flow statistics will be quantified. We examine the predictions for $c_{s}^{(\Delta)}$ from both the scale-invariant and the scale-dependent dynamic model in a numerical framework. Through comparison of the results to KPM04, the applicability of a priori results from field experiments to a posteriori settings in LES can be 
evaluated. Note that in HATS the filter size was defined in terms of the horizontal filter scale $\Delta_{h}$, namely $\Delta_{h} \equiv \Delta_{x}=\Delta_{y}$, where $\Delta_{x}$ and $\Delta_{y}$ are the filter sizes in the streamwise and spanwise directions, respectively. $\Delta_{x}$ and $\Delta_{y}$ also denote the horizontal grid spacings used in the LES of this paper. Furthermore, in the LES, the basic length scale used in the definition of eddy viscosity (e.g., equation (2)) is $\Delta=$ $\left(\Delta_{x} \Delta_{y} \Delta_{z}\right)^{1 / 3}=\left(\Delta_{h}^{2} \Delta_{z}\right)^{1 / 3}$ [Deardorff, 1974; Scotti et al., 1993], where $\Delta_{z}$ denotes the vertical grid size used in the LES. However, for consistency with the HATS experimental data, in this paper the results will be presented in terms of the horizontal filter scale $\Delta_{h}$ throughout. In LES, a horizontal cutoff filter is used in wave number space and implicit filtering by the grid spacing is assumed in the vertical. The variables used in the dynamic procedure for determination of the Smagorinsky coefficient (equation (3)) are filtered at $\alpha \Delta$ in the horizontal directions only, both in LES and HATS.

[9] During HATS, turbulence data were collected from two horizontal crosswind arrays of three-dimensional sonic anemometer-thermometers in the atmospheric surface layer. From the field data the empirically determined Smagorinsky model coefficient $c_{S}^{(\Delta, \text { emp })}$ was obtained by matching mean measured and modeled SGS dissipations $\Pi_{\Delta}$ [Clark et al., 1979]

$$
\left(c_{s}^{(\Delta, \mathrm{emp})}\right)^{2}=-\frac{\left\langle\tau_{i j} \widetilde{S}_{i j}\right\rangle}{\left\langle 2 \Delta_{h}^{2}\left|\widetilde{S}_{\mid}\right| \widetilde{S}_{i j} \widetilde{S}_{i j}\right\rangle}
$$

where the angle brackets denote Eulerian time averaging over a timescale $T_{c}$. Using this technique, Kleissl et al. [2003, hereinafter referred to as KMP03] and Sullivan et al. [2003] quantified the dependence of $c_{s}^{(\Delta)}$ upon distance to the ground and atmospheric stability. Specifically, KMP03 found that independently of $T_{c}$, the median of $c_{s}^{(\Delta)}$ is well described as a function of stability and height by an empirical fit:

$$
c_{s}^{(\Delta, \mathrm{emp})}=c_{0}\left[1+R\left(\frac{\Delta_{h}}{L}\right)\right]^{-1}\left[1+\left(\frac{c_{0}}{\kappa} \frac{\Delta_{h}}{z}\right)^{n}\right]^{-1 / n},
$$

where $R$ is the ramp function, $n=3, c_{0} \approx 0.135, L$ is the Obukhov length, and $\kappa$ is the van Karman constant. Using the same data set, KPM04 examined the ability of dynamic SGS models to predict the measured $c_{S}^{(\Delta, \mathrm{emp})}$ and its trends. Using the standard scale-invariant dynamic model it was found that the scale invariance assumption is violated when the filter size is large $\left(\Delta_{h}>z\right.$ or $\left.\Delta_{h}>L\right)$ resulting in coefficients that are too small. Conversely, the scaledependent dynamic model allows for scale dependence of the coefficient and as a result the predicted coefficients were found to be close to the measured values under various stability conditions. The objective of the present work is to compare the performance of the two versions of the dynamic model in LES (a posteriori).

[10] One important difference between the experimental analysis and the present simulations is the type of averaging employed to measure the coefficients: In the a priori analysis of KPM04, Eulerian time averaging over times $T_{c}$ was performed, whereas in the simulations time averaging along fluid path lines (Lagrangian averaging [Meneveau et al., 1996]) is used. Lagrangian time averaging was introduced for the general applicability of dynamic models to flows in complex geometries which do not possess spatial directions of statistical homogeneity over which to average [Bou-Zeid et al., 2004, 2005].

[11] This paper is organized as follows: The LES code and the Lagrangian SGS model are briefly described in section 2. Two test cases in stable and unstable conditions are analyzed in section 3 . Predictions for $c_{s}^{(\Delta)}$ from the simulation of a diurnal cycle are compared to HATS results in section 4 (a more detailed analysis of a diurnal simulation is presented by Kumar et al. [2006]). Conclusions follow in section 5 .

\section{Numerical Simulations}

\subsection{LES Code and Boundary Conditions}

[12] The conditions for the numerical simulations are selected to closely match the measurement conditions during HATS. Simulations are performed using a $160^{3}$ grid staggered in the vertical, and spanning a physical domain of $4000 \mathrm{~m} \times 4000 \mathrm{~m} \times 2000 \mathrm{~m}$, that is $\Delta_{x}=\Delta_{y}=25 \mathrm{~m}$, and $\Delta_{z}$ $=13 \mathrm{~m}$. The filtered Navier-Stokes equations are integrated over time based on the numerical approach described by Albertson and Parlange [1999a, 1999b].

$$
\partial_{i} \widetilde{u}_{i}=0
$$

$$
\begin{gathered}
\partial_{t} \widetilde{u}_{i}+\widetilde{u}_{j}\left(\partial_{j} \widetilde{u}_{i}-\partial_{i} \widetilde{u}_{j}\right)=-\partial_{i} \widetilde{p}^{*}-g \frac{\widetilde{\theta}^{\prime}}{\theta_{0}} \delta_{i 3}-\partial_{j} \tau_{i j} \\
+f\left(\widetilde{u}_{2}-v_{g}\right) \delta_{i 1}+f\left(u_{g}-\widetilde{u}_{1}\right) \delta_{i 2} \\
\partial_{t} \widetilde{\theta}+\partial_{j}\left(\widetilde{\theta} \widetilde{u}_{j}\right)=-\partial_{j} q_{j}
\end{gathered}
$$

The variable $\widetilde{\theta}^{\prime}=\widetilde{\theta}-\langle\widetilde{\theta}\rangle_{x, y}$ describes temperature fluctuations away from the planar averaged mean, $g$ is the gravitational acceleration, and $f$ is the Coriolis parameter. $q_{j}$ is the SGS heat flux

$$
q_{i}=-\operatorname{Pr}_{\mathrm{SGS}}^{-1} c_{s}^{2} \Delta^{2}|\widetilde{S}| \frac{\partial \widetilde{\theta}}{\partial x_{i}}
$$

where $P r_{\mathrm{SGS}}$ is the turbulent SGS Prandtl number, which is set to $P r_{\mathrm{SGS}}=0.4$. This is a value often used for neutral conditions [Kang and Meneveau, 2002, Figure 9b]. While $\operatorname{Pr}_{\mathrm{SGS}}$ depends on stability, it does not vary as much as $c_{s}$. Thus, in this work we prefer to focus on dynamic determination of $c_{s}$ while keeping $\operatorname{Pr}_{\mathrm{SGS}}$ fixed to avoid additional computational cost. For dynamic implementations of the SGS model for heat flux, see Porté-Agel [2004] and Stoll and Porté-Agel [2006].

[13] Pseudospectral discretization is used in horizontal planes and second-order finite differencing is implemented in the vertical direction. The second-order-accurate AdamBashforth scheme is used for time integration. Nonlinear convective terms and the SGS stress are dealiased using the 3/2 rule [Orszag, 1970]. Message passing interface (MPI) was implemented to run the simulation in parallel mode on supercomputers. 
[14] As in equation (2), $\Delta$ in equation (18) is defined as $\Delta$ $=\left(\Delta_{x} \Delta_{y} \Delta_{z}\right)^{1 / 3}$, while results will be reported as function of $\Delta_{h}$. The Coriolis parameter $f=\sin \Phi \times 1.45 \times 10^{-4} \mathrm{~s}^{-1}$ is imposed, using $\Phi \sim 36^{\circ} \mathrm{N}$ for the latitude of the HATS array. The modified pressure is $\widetilde{p}^{*}=\widetilde{p} / \rho_{0}+\frac{1}{3} \tau_{k k}+\frac{1}{2} \widetilde{u}_{j} \widetilde{u}_{j}$. $\left(u_{g}, v_{g}\right)$ are the components of the imposed geostrophic wind velocity.

[15] The horizontal boundary conditions are periodic and the vertical boundary conditions are zero vertical velocity and imposed stress at the bottom, and zero stress and zero vertical velocity at the top. The surface shear stresses are prescribed using Monin-Obukhov similarity law:

$$
\begin{gathered}
\tau_{13}=-\left(\frac{\kappa}{\ln z / z_{o}-\psi_{m}}\right)^{2}\left(\overline{\widetilde{u}}^{2}+\overline{\widetilde{v}}^{2}\right)^{0.5} \overline{\widetilde{u}} \\
\tau_{23}=-\left(\frac{\kappa}{\ln z / z_{o}-\psi_{m}}\right)^{2}\left(\overline{\widetilde{u}}^{2}+\overline{\widetilde{v}}^{2}\right)^{0.5} \overline{\widetilde{v}}
\end{gathered}
$$

where $\overline{\widetilde{(})}$ represents a local average from filtering the velocity field at $2 \Delta$ (see Bou-Zeid et al. [2005] for more details about the need for such filtering). The roughness length at the surface is set to $z_{o}=0.02 \mathrm{~m}$, equivalent to the value determined from the HATS data, and van Karman's constant $\kappa=0.4$. The flux profile functions in unstable conditions are given by Dyer [1974] with the correction by Hogstrom [1987], while in stable conditions we use the formulation by Brutsaert [2005]:

$$
\begin{gathered}
\phi_{m}=(1-15.2 z / L)^{-1 / 4} \text { when } L<0 \\
\phi_{m}=1+6.1 \frac{z / L+(z / L)^{a}\left(1+(z / L)^{a}\right)^{-1+1 / a}}{z / L+\left(1+(z / L)^{a}\right)^{1 / a}} \text { when } L>0 \\
\phi_{h}=(1-15.2 z / L)^{-1 / 2} \text { when } L<0 \\
\phi_{h}=1+5.3 \frac{z / L+(z / L)^{b}\left(1+(z / L)^{b}\right)^{-1+1 / b}}{z / L+\left(1+(z / L)^{b}\right)^{1 / b}} \text { when } L>0
\end{gathered}
$$

where $a=2.5$ and $b=1.1$.

[16] The $\psi_{m}$ functions are determined as follows:

$$
\psi_{m}(z / L)=\int_{z_{o} / L}^{z / L}\left[1-\phi_{m}(x)\right] d x / x .
$$

[17] These wall models are themselves parameterizations for unresolved near-surface fluxes occurring at scales below the first grid point and involve a series of modeling uncertainties. For a discussion, see, for example, Piomelli and Balaras [2002].

[18] Near the top boundary of the domain, a numerical sponge is applied to dissipate energy of gravity waves
Table 1. Details of the Four Simulations Conducted for This Study ${ }^{\mathrm{a}}$

\begin{tabular}{lccccc}
\hline & \multicolumn{2}{c}{ Unstable } & & \multicolumn{2}{c}{ Stable } \\
\cline { 2 - 3 } \cline { 5 - 6 } \multicolumn{1}{c}{ Parameter } & DYN & SD & & DYN & SD \\
\hline$\left\langle w^{\prime} \theta^{\prime}\right\rangle_{s}, \mathrm{Kms}^{-1}$ & 0.1 & 0.1 & & -0.02 & -0.02 \\
$t_{\text {avg }}, \mathrm{h}$ & $3-4$ & $3-4$ & & $10-12$ & $10-12$ \\
$z_{i}, \mathrm{~m}$ & 855 & 855 & & 212 & 162 \\
$L, \mathrm{~m}$ & -43 & -42 & & 61 & 45 \\
\hline
\end{tabular}

${ }^{\mathrm{a}}$ All simulations were conducted in a domain of $4000 \times 4000 \times 2000 \mathrm{~m}$ and at a resolution of $160^{3}$. "DYN" abbreviates the Lagrangian scaleinvariant dynamic simulation, while "SD" abbreviates the Lagrangian scale-dependent dynamic simulation. The time period of the simulation used for the quantitative analysis is given by $t_{\text {avg }}$. The inversion height $z_{i}$ was determined as the location of minimum heat flux for the unstable simulations and as the location where the momentum flux is $5 \%$ of its surface value in the stable simulations.

before they reach the upper boundary of the domain [Nieuwstadt et al., 1991]. The sponge treatment is applied to the four uppermost levels of the grid. The simulations are forced with prescribed geostrophic velocity $\left(u_{g}, v_{g}\right)$ and surface kinematic heat flux $\left\langle w^{\prime} \theta^{\prime}\right\rangle_{s}$. The boundary layer height, $z_{i}$, is used as a characteristic length scale.

\subsection{Lagrangian Scale-Dependent Dynamic SGS Model}

[19] In LES with the dynamic model, the numerator and denominator in equation (8) need to be averaged over homogeneous areas or over time in order to prevent negative eddy viscosities that may lead to numerical instabilities. Typically in channel flow, or ABL flow, $c_{s}^{(\Delta)}$ is computed from quantities averaged over horizontal planes. Though spatial averaging across horizontal planes in flow over heterogeneous surfaces is not appropriate, time averaging is always possible in principle. However, to comply with Galilean invariance, time averaging must be performed following material fluid elements, and this leads to the development of the Lagrangian dynamic model [Meneveau et al., 1996].

[20] The original Lagrangian SGS model uses the definition of equation (5) with $\beta=1$ (that is the scaleinvariant version). As discussed previously, this assumption leads to inaccurate results when $\Delta$ approaches the limits of an idealized inertial range of turbulence. To remedy this, a scale-dependent dynamic version of the Lagrangian SGS model is also used in the simulations. For detailed information on the implementation, see Bou-Zeid et al. [2005].

\section{Unstable and Stable Test Cases}

[21] The LES model using the Lagrangian scale-dependent dynamic model gives excellent results in neutral conditions [Bou-Zeid et al., 2005]. Nondimensional velocity gradients and velocity energy spectra confirm well known experimental results such as the $k^{-5 / 3}$ scaling in the inertial range, a nearly $k^{-1}$ in the production range close to the ground, and normalized mean velocity profiles $\Phi_{m}=$ $\kappa z u_{*}^{-1} \partial\left\langle u_{1}\right\rangle / \partial z \approx 1$ in the neutral surface layer [Parlange and Brutsaert, 1989]. To study the effects of stability and the choice of SGS model on the dynamic Smagorinsky coefficient, four $160^{3} \mathrm{LES}$ with constant surface heat fluxes are performed using scale-invariant $(\beta=1)$ and scale- 

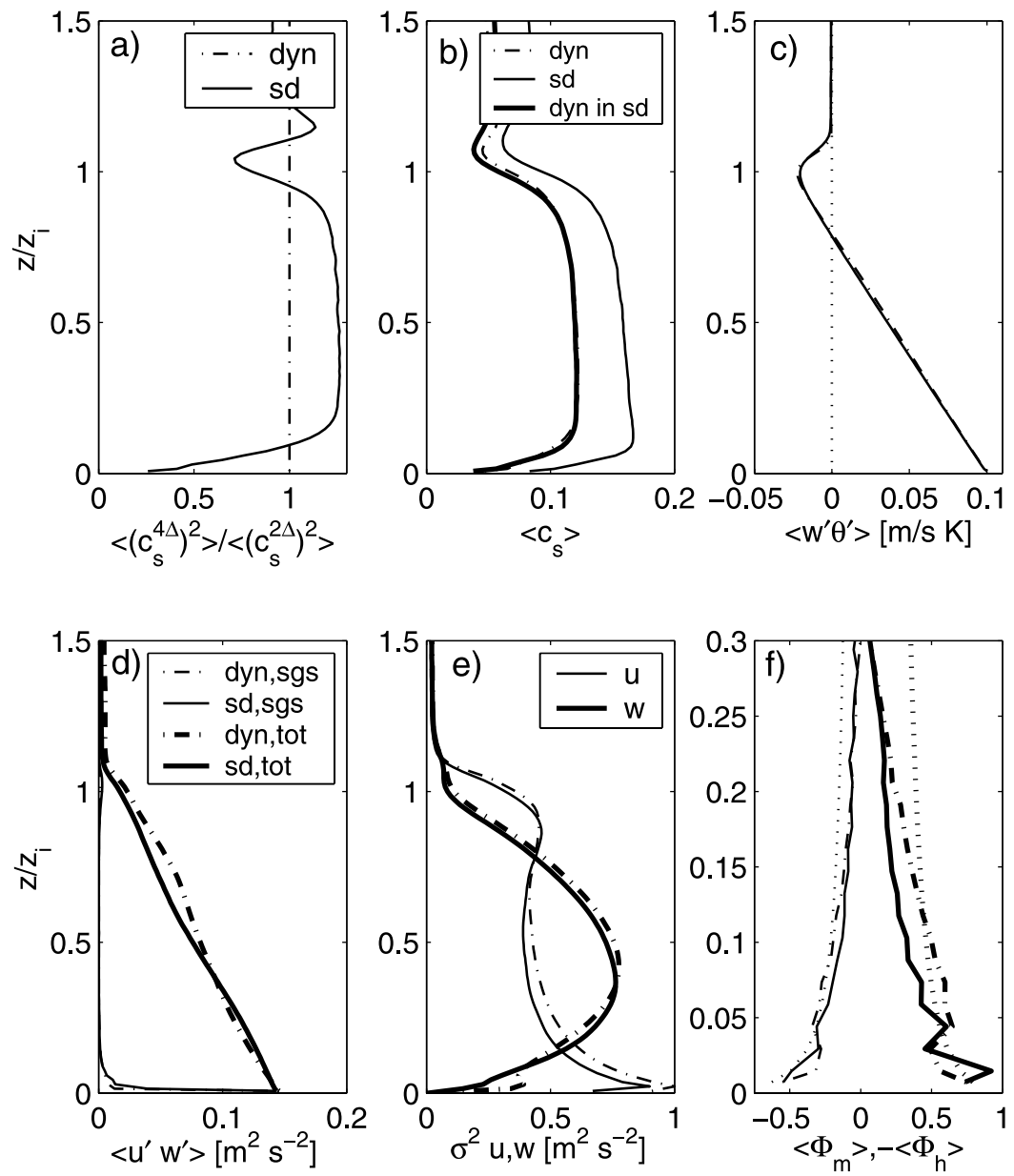

Figure 1. Profiles of quantities averaged over 1 hour during LES with $\left\langle w^{\prime} \theta^{\prime}\right\rangle_{s}=0.1 \mathrm{~K} \mathrm{~m} \mathrm{~s}^{-1}$. Dotdashed lines are results using the scale-invariant version of the dynamic subgrid model; solid lines are results using the scale-dependent version. (a) Scale dependence parameterized as $\left\langle\left(c_{s}^{4 \Delta}\right)^{2}\right\rangle /\left\langle\left(c_{s}^{2 \Delta}\right)^{2}\right\rangle$ and (b) Smagorinsky coefficient $c_{s}^{(\Delta)}$. The Smagorinsky coefficient derived from the scale-invariant procedure applied to the velocity field of the scale-dependent dynamic simulation is shown as a thick line. (c) Total vertical heat flux $\left\langle\widetilde{w}^{\prime} \widetilde{\theta}^{\prime}\right\rangle+q_{3}$, (d) SGS $\left(\tau_{13}^{2}+\tau_{23}^{2}\right)^{0.5}$ and total resulting horizontal shear stress $\left[\left(\left\langle\widetilde{u}^{\prime} \widetilde{w}^{\prime}\right\rangle+\tau_{13}\right)^{2}+\left(\left\langle\widetilde{v}^{\prime} \widetilde{w}^{\prime}\right\rangle+\tau_{23}\right)^{2}\right]^{0.5}$, (e) resolved velocity variances $\sigma^{2}(\widetilde{u})$ and $\sigma^{2}(\widetilde{w})$, and (f) nondimensional velocity gradient $\Phi_{m}=\kappa z u_{*}^{-1} \partial \widetilde{u} / \partial z$ (thin curves) and nondimensional temperature gradient $\Phi_{h}=-\kappa z u * /\left\langle\widetilde{w}^{\prime} \theta^{\prime}\right\rangle \partial \theta / \partial z$ (thick curves). For comparison, the empirical surface layer functions (equation (22)) are shown as dotted lines.

dependent $(\beta \neq 1)$ SGS parameterizations. Table 1 shows an overview of the simulations. In the unstable simulation, the surface heat flux is $\left\langle w^{\prime} \theta^{\prime}\right\rangle_{s}=0.1 \mathrm{Kms}^{-1}$ and the results are averaged over the last hour of a four hour simulation. In the stable simulation, the surface heat flux is $\left\langle w^{\prime} \theta^{\prime}\right\rangle_{s}=-0.02$ $\mathrm{Kms}^{-1}$ and the results are averaged over the last two hours of a twelve hour simulation. The simulations are initialized with a constant mean temperature profile below $800 \mathrm{~m}$ and an inversion layer of strength $0.01 \mathrm{Km}^{-1}$ above $800 \mathrm{~m}$ to limit the vertical growth of the boundary layer in unstable conditions. The geostrophic velocity is $\left(u_{g}, v_{g}\right)=(8,0) \mathrm{m} \mathrm{s}^{-1}$.

\subsection{Simulations for Unstable Conditions}

[22] Vertical profiles for the simulations of unstable conditions for both models are shown in Figure 1. The stability parameter $L \sim-42 \mathrm{~m}\left(\Delta_{h} / L \sim-0.60\right)$ indicates unstable conditions. The height of the capping inversion $z_{i}$ is often defined as the location of minimum heat flux (Figure 1c). This occurs at $z_{i} \sim 855 \mathrm{~m}$ for both simulations.
In general, the results for the scale-invariant and scaledependent SGS models are quite similar. In unstable simulations at high resolution, the SGS do not contain much energy. Thus the SGS model's influence on the profiles of mean quantities, variances, and covariances is limited, except near the land surface.

[23] In Figure 1a it can be seen that in stable conditions and near the surface, the Smagorinsky coefficient becomes scale-dependent in the SD simulation. Note that since averages of $\beta$ are not meaningful due to occasional large values when the denominator of equation (12) is very small, we use the average squared coefficient at $4 \Delta$ divided by the average squared coefficient at $2 \Delta$ as a measure of scale dependence. This measure is about 1.2 in the mixed layer and decreases to 0.3 near the surface indicating that the scale dependence of $c_{S}$ is stronger near the surface, causing an increase in $c_{s}^{(\Delta)}$ as compared to the DYN simulation (Figure 1b). In the mixed layer, $c_{s}^{(\Delta)} \sim 0.16$ in the SD simulation, while $c_{s}^{(\Delta)} \sim 0.11$ in the DYN simulation. To 

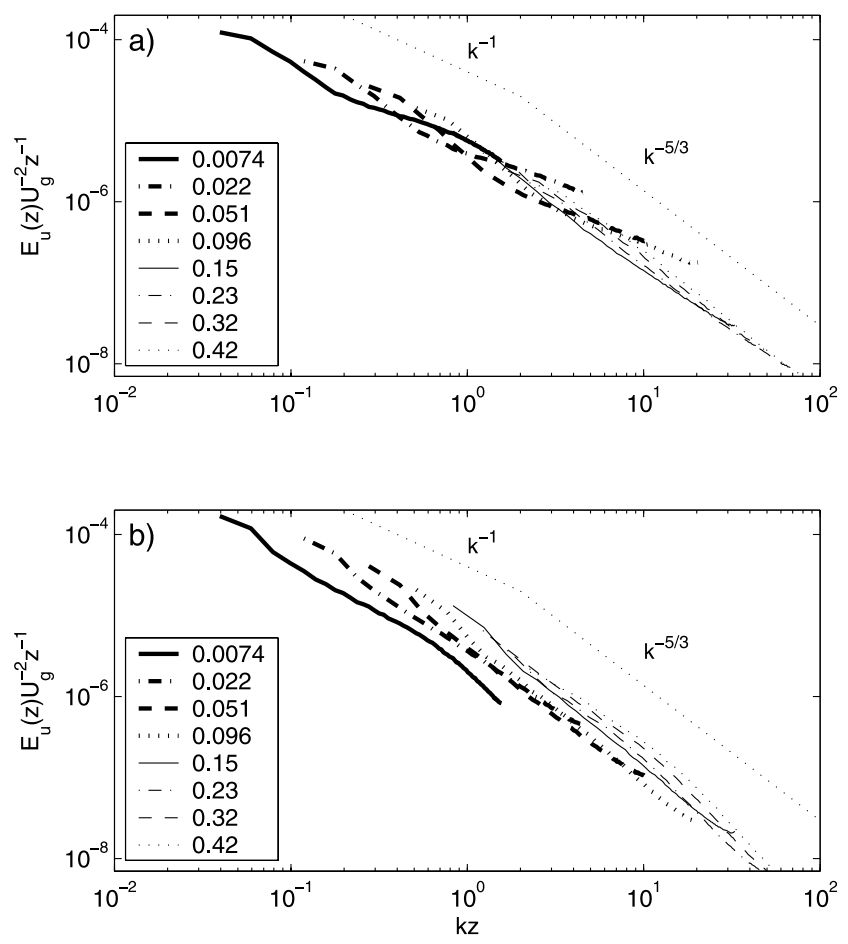

Figure 2. Normalized streamwise velocity power spectra versus $k z$ at different heights for unstable conditions. Heights $z / z_{i}$ are given in the legend. (a) Standard Lagrangian dynamic SGS model and (b) scale-dependent Lagrangian dynamic SGS model.

examine how the difference in the velocity fields between the two simulations influences the value of $c_{s}$, the scaleinvariant dynamic model was applied to the velocity field of the scale-dependent dynamic model (without using the resulting coefficient in the simulation). Figure $1 \mathrm{~b}$ indicates that the Smagorinsky coefficient derived from the scaleinvariant dynamic model is too small, even if derived from the SD simulation. Note that the Lagrangian SGS model used in this simulation [Bou-Zeid et al., 2005] assumes that the scale-invariant dynamic model gives a correct estimate for $c_{s}$ at the test filter scale. For the neutral simulation, the self-consistency of this assumption was tested by plotting the results from the scale-invariant model as function of $z$ / $2 \Delta$ (height normalized with test filter scale) and comparing with the scale-dependent model plotted as function of $z / \Delta$, and finding good collapse (POR). In the present case with thermal effects affecting the scale dependence, it is less obvious how to perform such an intercomparison. At any rate, the trends as function of normalized height are similar as those in POR.

[24] In the stable region above the capping inversion at $855 \mathrm{~m}, c_{s}^{(\Delta)}$ decreases and reaches a value of $c_{s} \sim 0.08$ and $c_{s} \sim 0.05$ for the SD and DYN model, respectively. Above the inversion height, the turbulent stresses and variances are close to zero. For both SGS models, shear stress (Figure 1d) and velocity variance (Figure 1e) profiles are qualitatively similar to previous results for LES of convective boundary layers [e.g., Moeng and Sullivan, 1994]. The nondimensional velocity gradient $\Phi_{m}$ and temperature gradient $\Phi_{h}$ are shown in Figure 1f. As expected, they follow empirical functions (equation (21)) in the surface layer $(z<150 \mathrm{~m})$, although some oscillations near the surface are observed.

[25] While the correct representation of the mean profiles by the SGS model is important, better information on the correct representation of turbulent structures can be obtained from the velocity spectra. For unstable conditions, but shear-dominated flow (as in the surface layer) one would expect to see $a-1$ scaling in the production range (large scales) and an inertial subrange with a $-5 / 3$ power law. In buoyancy-dominated flow (e.g., above a height equal to the Obukhov length) the inertial subrange extends to smaller wave numbers and the -1 power law in the production range may not be observed [Stull, 1997]. Figure 2 shows the streamwise velocity spectra for the DYN and SD simulations. In the near-surface region $\left(z / z_{i}<0.1\right)$ which is the most challenging for a SGS model, the spectra in the SD simulation agree very well with the inertial subrange scaling of $k^{-5 / 3}$, while the spectra for the DYN simulations are too flat. This reflects the underdissipative property of the scaleinvariant dynamic model near the wall already noted in POR. At greater heights in the mixed layer the turbulence spectra are consistent with the inertial range scaling for both SGS models. The temperature spectra in Figure 3 lead to similar conclusions.

[26] In summary, while both simulations show similar mean profiles, the scale-dependent dynamic model represents the energy transfer between resolved and unresolved turbulence structures more accurately as reflected in the power spectra. Since the SGS represent a greater amount of TKE in stable atmospheric conditions, a more conclusive test for SGS models will be presented in the next section using stable simulations.

\subsection{Stable Simulations}

[27] While the unstable boundary layer grows steadily into the inversion region, the stable boundary layer is
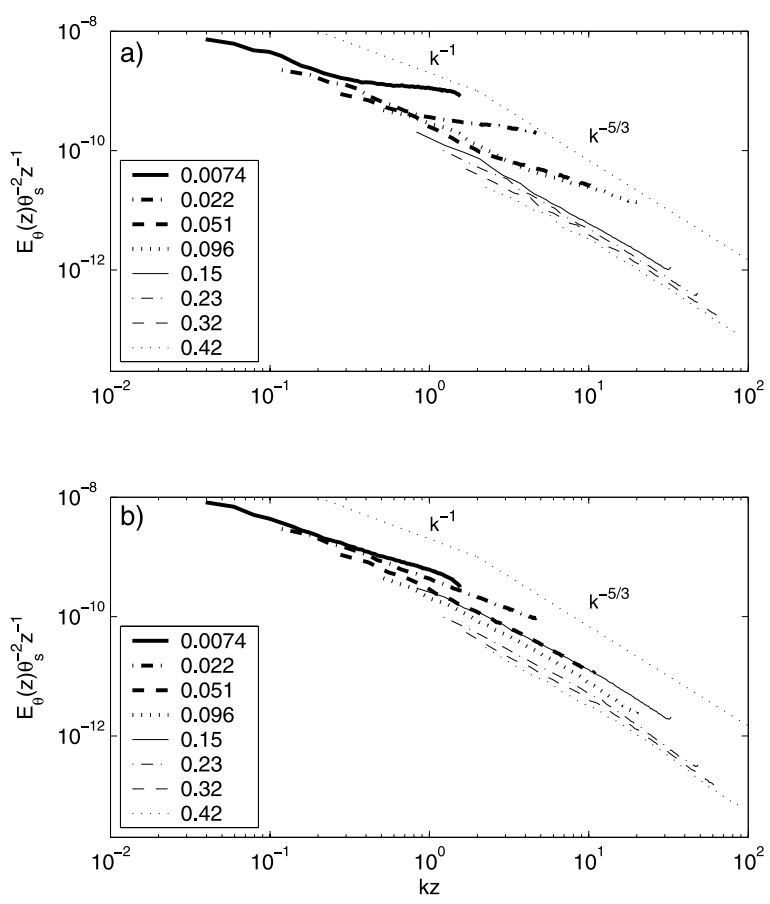

Figure 3. Same as Figure 2 for temperature power spectra. 

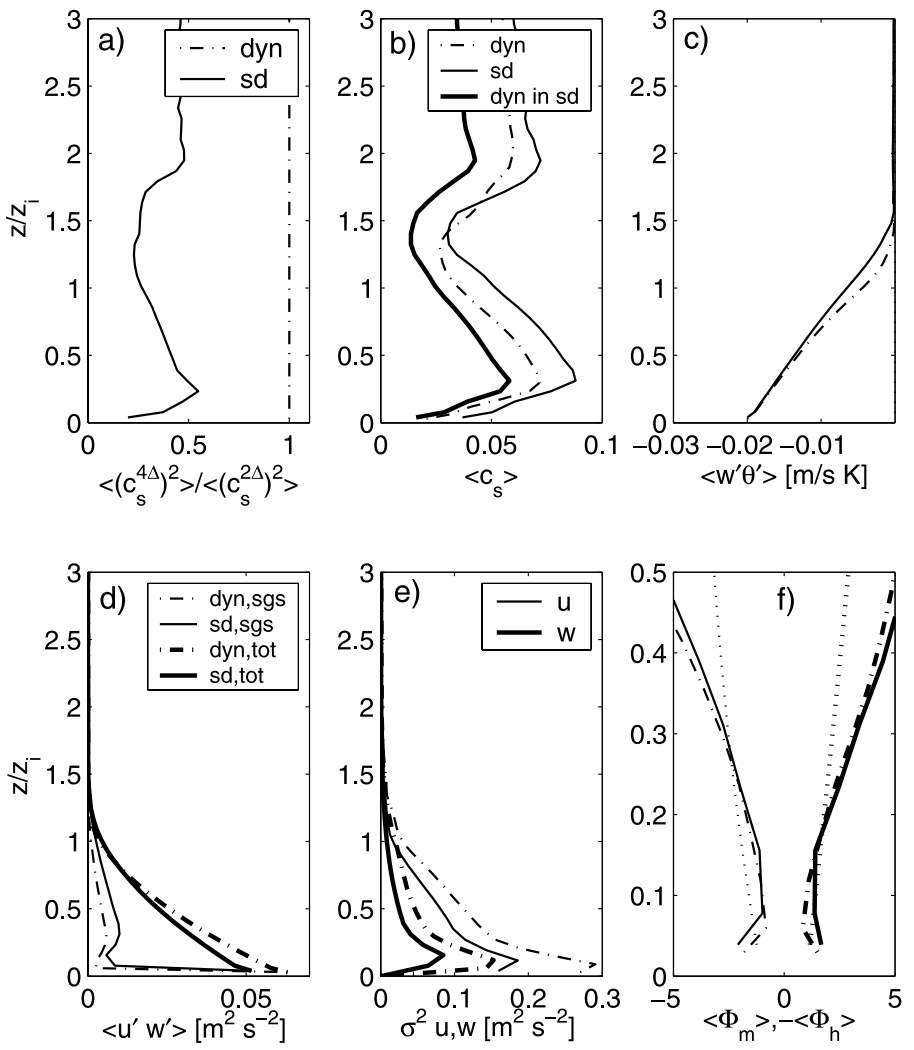

Figure 4. Profiles of quantities averaged over 2 hours during a LES with $\left\langle w^{\prime} \theta^{\prime}\right\rangle_{s}=-0.02 \mathrm{~K} \mathrm{~m} \mathrm{~s}^{-1}$. Dotdashed lines are results using the scale-invariant version of the model; solid lines are results using the scale-dependent version. (a) Scale dependence parameterized as $\left\langle\left(c_{s}^{4 \Delta}\right)^{2}\right\rangle /\left\langle\left(c_{s}^{2 \Delta}\right)^{2}\right\rangle$ and (b) Smagorinsky coefficient $c_{s}^{(\Delta)}$. (c) Total vertical heat flux $\left\langle\widetilde{w}^{\prime} \theta^{\prime}\right\rangle+q_{3}$, (d) SGS $\left(\tau_{13}^{2}+\tau_{23}^{2}\right)^{0.5}$ and total resulting horizontal shear stress $\left[\left(\left\langle\widetilde{u}^{\prime} \widetilde{w}^{\prime}\right\rangle+\tau_{13}\right)^{2}+\left(\left\langle\widetilde{v}^{\prime} \widetilde{w}^{\prime}\right\rangle+\tau_{23}\right)^{2}\right]^{0.5}$, (e) resolved velocity variances $\sigma^{2}(\widetilde{u})$ and $\sigma^{2}(\widetilde{w})$, and (f) nondimensional velocity gradient $\Phi_{m}=\kappa z u_{*}^{-1} \partial \widetilde{u} / \partial z$ (thin curves) and nondimensional temperature gradient $\Phi_{h}=-\kappa z u_{*} /\left\langle\widetilde{w}^{\prime} \bar{\theta}^{\prime}\right\rangle \partial \theta / \partial z$ (thick curves). For comparison, the empirical surface layer functions (equation (21)) are shown as dotted lines.

shallow and largely unaffected by the inversion region. Therefore, in Figures $4 \mathrm{a}-4 \mathrm{f}$ only the lower half of the simulation domain is presented. To reach quasi-steady conditions [Kosović and Curry, 2000], the simulation with $\left\langle w^{\prime} \theta^{\prime}\right\rangle_{s}=-0.02 \mathrm{Kms}^{-1}$ was run for a physical duration of 10 hours. Subsequently, averages were calculated over the following 2 hours. The Obukhov length was $L \sim 61 \mathrm{~m}$ $\left(\Delta_{h} / L \sim 0.41\right)$ in the DYN simulation and $L \sim 45 \mathrm{~m}\left(\Delta_{h} /\right.$ $L \sim 0.56)$ in the SD simulation, characterizing moderately stable conditions. Note that overall the Smagorinsky coefficients in the stable simulation were significantly smaller than in the unstable runs. Heat fluxes (Figure 4c), stresses (Figure 4d), and variances (Figure 4e) decreased to zero at $z \sim 200 \mathrm{~m}$, indicating the height of the stable boundary layer. The stable boundary layer height $z_{i}$ was defined as the location where the shear stresses reach $5 \%$ of their surface value (see Table 1). In contrast to the unstable simulations, here the mean profiles from the SD and DYN simulations are markedly different. In stable boundary layers, the SGS contain a significant amount of the total turbulence kinetic energy [Beare et al., 2006]. Thus the quality of the SGS model will have a greater influence on the overall simulation results.

[28] The most important distinction is that the stable boundary layer has grown higher in the DYN simulation than in the SD simulation. This is expected, since the reduction in turbulence kinetic energy due to the larger $c_{s}^{(\Delta)}$ in the scale-dependent model leads to a slower growth of the stable boundary layer. Boundary layer growth has been identified as a key parameter in a stable LES intercomparison study [Beare et al., 2006]. However, even the profiles normalized by $z_{i}$ do not collapse, indicating a fundamental difference between the results of the two simulations.

[29] The velocity variances, stresses, and heat flux were larger in the DYN simulation, indicating the underdissipative property of this SGS model. As in the unstable simulations, the decreased $\beta$ in the SD simulation causes $c_{s}^{(\Delta)}$ to increase as compared to the DYN simulation (Figure 4b). However, the Smagorinsky coefficient determined by applying the scale-invariant SGS model to the velocity field in the SD simulation does not agree with the $c_{s}^{(\Delta)}$ profile in the DYN simulation. This is mainly due to the different boundary layer profiles which developed over the 12 hour simulation period. Despite these differences, the nondimensional velocity and temperature profiles are similar in both simulations, and agree well with empirical profiles below $z \sim 50 \mathrm{~m}$.

[30] Further clues on the representation of turbulence structures in the simulations are obtained from the stream- 

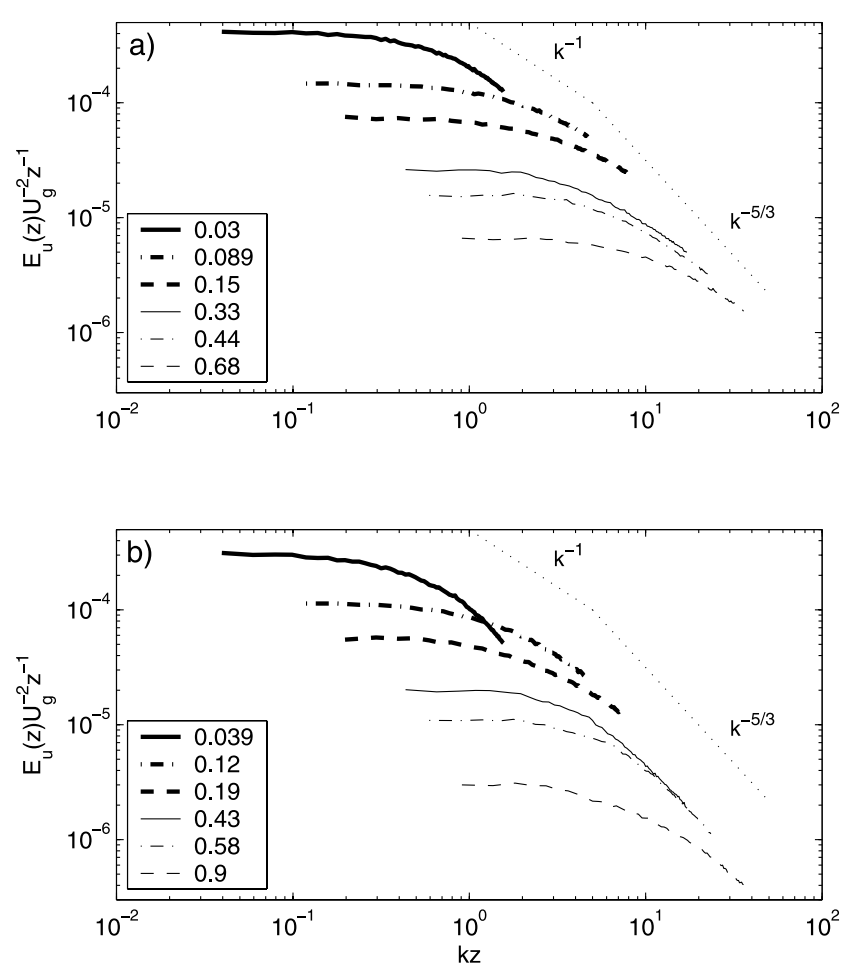

Figure 5. Normalized streamwise velocity power spectra versus $k z$ at different heights for stable conditions. Heights $z / z_{i}$ are given in the legend. (a) Standard Lagrangian dynamic SGS model and (b) scale-dependent Lagrangian dynamic SGS model.

wise velocity spectra in Figure 5 and temperature spectra in Figure 6 . For the stable boundary layer, the -1 power law for large eddies in the production range may not be observable due to opposition to turbulent motions by stability (a $k^{-1}$ line is still included for reference). An inertial subrange with a $-5 / 3$ power law is still expected, but the lower wave number end becomes larger for increasing stability [Stull, 1997]. Similar to the results for unstable conditions, the spectra for the DYN simulations are flat, while those of the SD model are steeper, in general closer to the expected $k^{-5 / 3}$ scaling in the inertial range.

[31] In summary, we conclude that the LES with the Lagrangian scale-dependent dynamic SGS model captures the main features of stable and unstable boundary layers. The choice of SGS model does not influence the mean profiles in the unstable case, where the scale-dependent and scale-invariant models predict essentially similar mean velocity and temperature gradients. However, the velocity spectra in stable and unstable conditions indicate that the scale-dependent dynamic model represents the turbulence structures more faithfully.

\section{Smagorinsky Coefficient as a Function of $\Delta / L$ in a Diurnal Cycle of the ABL and Comparison to HATS}

[32] Here our goal is to compare the Smagorinsky coefficients obtained from the dynamic and scale-dependent dynamic models during the simulation of a diurnal cycle to HATS measurements. The HATS data set includes data from a wide range of stability conditions $\left(1<\Delta_{h} / L<10\right.$,
KMP03). The LES data set is based on the simulation presented in detail by Kumar et al. [2006], where it is suggested that under very stable conditions (typically $\Delta_{h} \gg L$ ), LES based on the Smagorinsky eddy viscosity parameterizations display instabilities, although the scaledependent dynamic model returns realistic coefficient values. For the purposes of the present paper, however, simulations are carried out in stability regimes under which the simulations do not display these instabilities. The simulation still created an evolution of stability conditions qualitatively and quantitatively similar to the experiment, except that the extremely stable conditions are not matched. The most stable conditions in our simulation were $L \sim 6.9 \mathrm{~m}, \Delta_{h} / L \sim 3.6$, and $z / L \sim 1.8$ at the first grid point.

[33] A plot of the evolution of $c_{s}^{(\Delta)}$ from the simulation with $\beta \neq 1$ as a function of time and height is shown in Figure 7a. The evolution of the Smagorinsky coefficient obtained by applying the scale-invariant procedure to the velocity field of the scale-dependent simulation is presented in Figure $7 b$.

[34] As observed in the experiment, the coefficient decreases near the wall and in stable stratification. Since the coefficient is derived from a mixing length assumption it can be interpreted as the ratio of an SGS turbulence length scale to the filter scale. In these conditions the observed decrease in $c_{S}$ could thus be interpreted as a decrease of the eddy sizes of the SGS turbulence when shear, wall blocking, or stratification are large.

[35] The coefficient decreases after sunset (1730h) and remains very small during stable conditions at night. Conversely, $c_{s}^{(\Delta)}$ increases in unstable daytime conditions. Above the daytime boundary layer, the stable capping inversion produces a smaller $c_{s}^{(\Delta)}$. During the evening transition, large $c_{s}^{(\Delta)}$ persist at mid-ABL heights $(\sim 500 \mathrm{~m})$
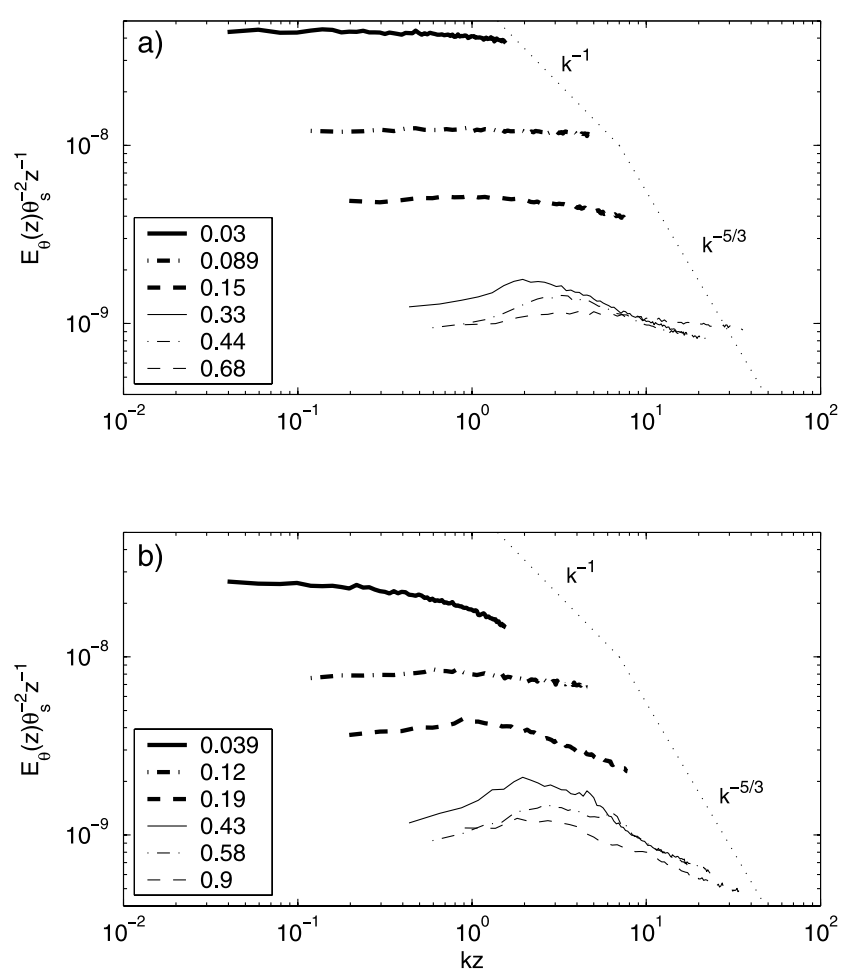

Figure 6. Same as Figure 5 for temperature power spectra. 

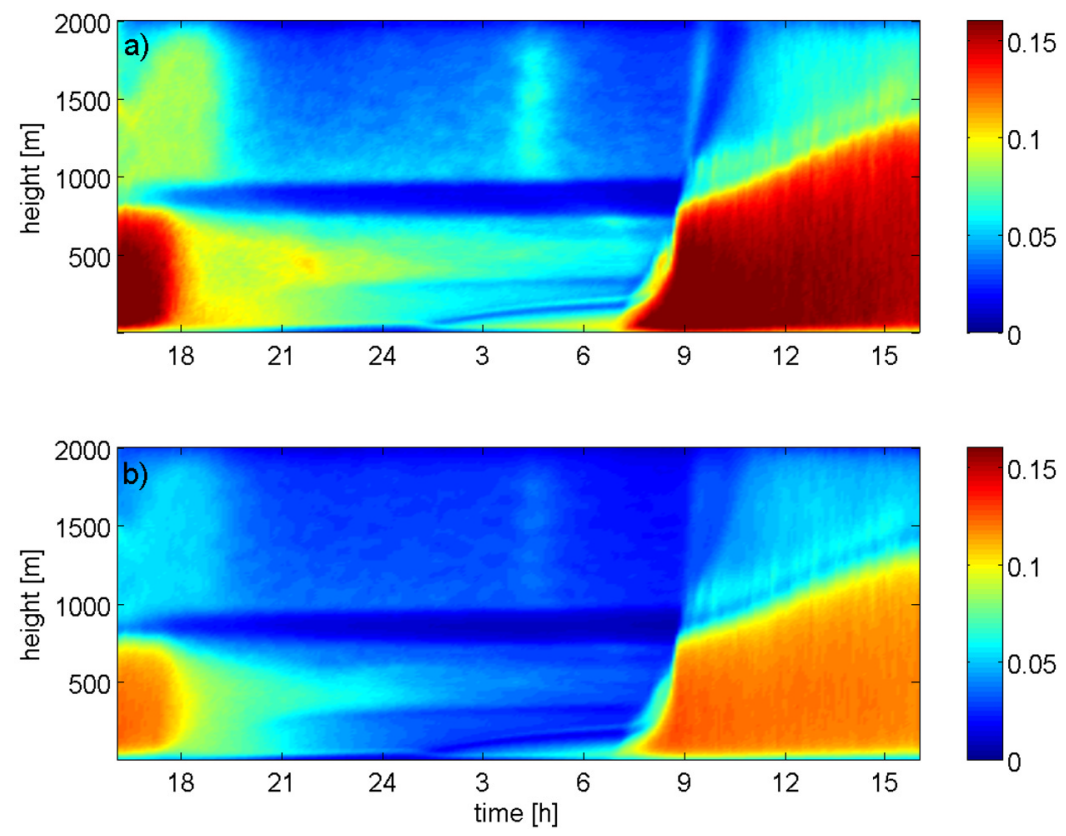

Figure 7. Daily evolution of $\left(c_{S}^{\Delta}\right)^{2}(z)$ averaged over $x$ and $y$. (a) Scale-dependent dynamic SGS model and (b) scale-invariant dynamic SGS model applied to the velocity field of the simulation with the scaledependent dynamic model.

until 2200h. During the morning transition, the first strong increase in $c_{s}^{(\Delta)}$ occurs near the surface at $0710 \mathrm{~h}, \sim 30 \mathrm{~min}$ after sunrise $(0640 \mathrm{~h})$. With the rapidly increasing ABL height, $c_{s}^{(\Delta)}$ also quickly increases at greater heights. Comparing to the coefficient obtained from the scale-invariant dynamic procedure, (Figure $7 \mathrm{~b}$ ) it is observed that the scale-dependent $c_{s}^{(\Delta)}$ is always significantly larger than the scale-invariant $c_{s}^{(\Delta)}$. The ratio of the scale-dependent and scaleinvariant $c_{s}^{(\Delta)}$ (not shown) is largest near the top of the stable boundary layer with a value of $\sim 2$, and in daytime near the surface and in the entrainment layer with a value of $\sim 1.5$. While $c_{s}^{(\Delta)}$ during the morning transition is similarly predicted by the two SGS models, the evening transition from large $c_{s}^{(\Delta)}$ to small $c_{s}^{(\Delta)}$ is prolonged when using the scale-dependent formulation. Larger Smagorinsky coefficients in the nocturnal boundary layer will result in slower boundary layer growth, as observed in section 3.2.

[36] Next, the LES results are compared to the HATS data fit (equation (14)) in Figure 8. While the LES predictions by both SGS models capture the decrease of $c_{S}^{(\Delta)}$ in stable conditions during HATS qualitatively, $c_{s}^{(\Delta)}$ from the scaleinvariant model is too small. The Smagorinsky coefficient computed from the scale-dependent procedure is closer to the value from the empirical fit. In unstable conditions, $c_{S}^{(\Delta)}$ continues to increase with increasingly unstable atmospheric conditions for both models, while the empirical formula is constant for $L<0$.

[37] The other important observation from Figure 8 is a delay in the response of the Smagorinsky coefficient to changing surface conditions at greater heights (smaller $\Delta_{h} / z$ ). In Figure $8 \mathrm{a}, \Delta_{h} / L$ collapses the data for $z=6.3 \mathrm{~m}\left(\Delta_{h} / z=4\right)$ reasonably well. At greater heights, however, two significantly different values are obtained for $c_{s}^{(\Delta)}$ depending on whether it is the morning or evening transition (hysteretic behavior observed in Figures $8 \mathrm{~b}$ and $8 \mathrm{c}$ ). This behavior is physically expected due to the following considerations: In
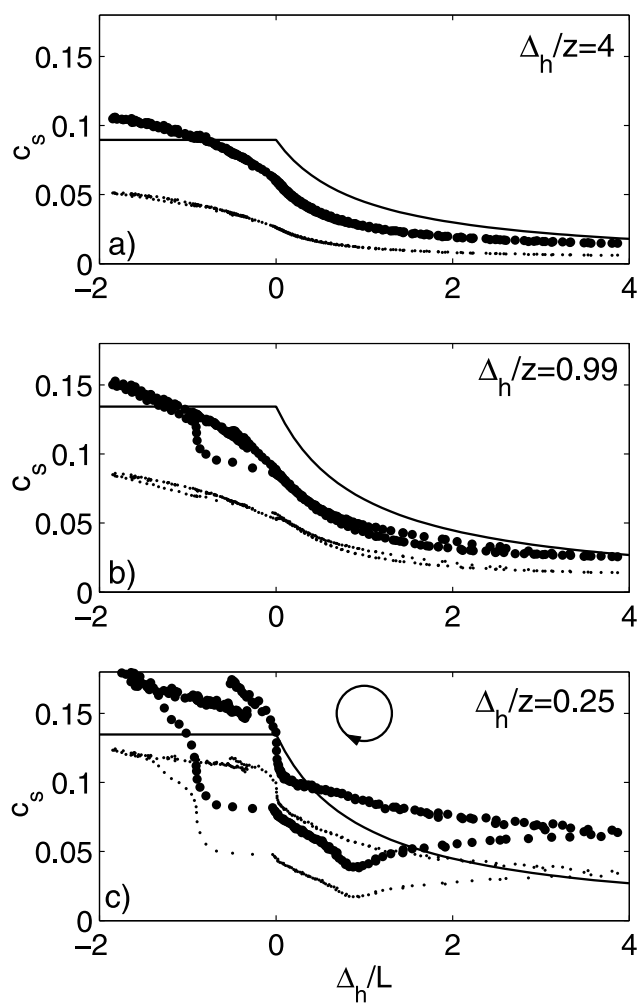

Figure 8. (a-c) Parameter $c_{S}^{\left({ }^{()}\right)}$as a function of $\Delta_{h} / L$ for three heights in the diurnal simulation. The circle with the clockwise arrow in Figure 8c indicates the sense of the time sequence. Large dots depict the Smagorinsky coefficient from the scale-dependent dynamic model. Small dots depict the Smagorinsky coefficient from the scale-invariant dynamic model. 

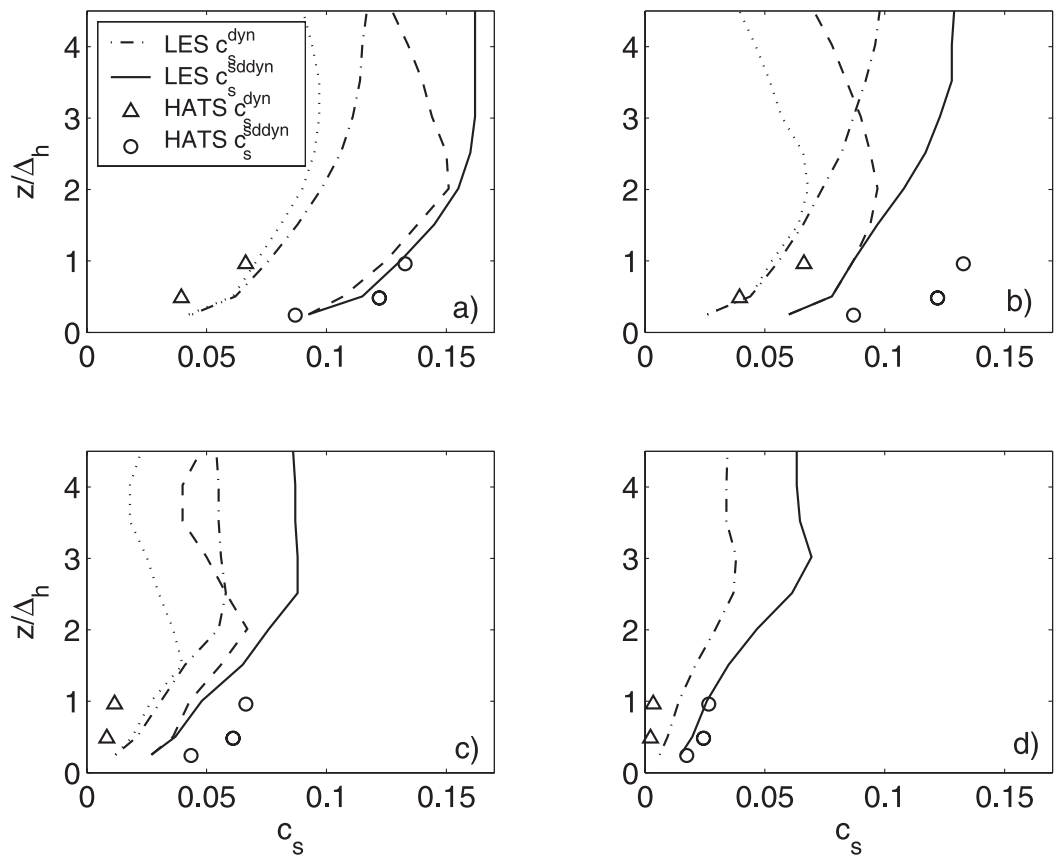

Figure 9. Smagorinsky coefficient $c_{S}^{(\Delta)}$ for different stability conditions from HATS and from LES. (a) $\Delta_{h} / L \sim-1$, (b) $\Delta_{h} / L \sim 0$, (c) $\Delta_{h} / L \sim 1$, and (d) $\Delta_{h} / L \sim 4$. Dot-dashed and dotted lines are scaleinvariant dynamic SGS model; solid and dashed lines are scale-dependent dynamic SGS model. Because of hysteretic behavior that is observed in the near-neutral stability, in Figures 9a, 9b, and 9c, two curves for each simulation are plotted.

the early morning the instability increases rapidly with time. Since it takes some time for the turbulence at a greater height to adjust to the new conditions at the surface, the stability conditions at greater heights are less unstable than those close to the surface. This difference is extreme at a height that is still outside of the turbulent boundary layer and thus dynamically disconnected from the unstable regime near the surface. Conversely, in the evening the stability conditions become slowly less unstable (decaying turbulence), and thus the turbulence has more time to adjust to changing surface conditions. It is expected that a change in surface conditions needs several large eddy turnover times $\left(\sim 100 \mathrm{~m} / u_{*} \sim 400 \mathrm{~s}\right)$ to affect the entire surface layer. The observed hysteretic behavior is examined in more detail by Kumar et al. [2006], who conclude that local scaling is successful in describing the behavior of the coefficient.

[38] In Figure 9 the predictions for the Smagorinsky coefficient from the simulations are compared to the measured coefficients from HATS described by KMP03 $\left(c_{s}^{(\Delta, \mathrm{emp})}\right)$ and the predicted dynamic coefficients from HATS of KPM04 $\left(c_{s}^{(\Delta \text {,dyn })}, c_{s}^{(\Delta \text {,sd-dyn })}\right)$. In experiment and simulation, the scale-dependent coefficient is always larger than the scale-invariant. In general, the scale-dependent coefficients from the simulation match the experimentally determined values $c_{S}^{(\Delta, \mathrm{emp})}$. In addition, the data from HATS and from LES agree well for the scale-invariant case $\left(c_{s}^{(\Delta, \text { dyn })}\right)$, although, as noted before, the values fall significantly below the measured coefficient $c_{s}^{(\Delta, \mathrm{emp})}$.

[39] The hysteretic behavior of the coefficient in Figure 8 has to be taken into account when plotting the results. Consequently in Figure 9 for $\Delta_{h} / L \sim-1$ in Figure 9a, $\Delta_{h} / L$ $\sim 0$ in Figure 9b, and $\Delta_{h} / L \sim 1$ in Figure 9c, two data sets are plotted for each of the simulations: The larger values are recorded during the evening transition. The smaller values occur during the morning transition, when as outlined earlier, $\Delta_{h} / L$ is not an appropriate scaling parameter.

[40] In the simulation, $c_{s}^{(\Delta)}$ is larger in unstable conditions (Figure 9a) than in neutral conditions (Figure 9b), in contrast to HATS results. The Smagorinsky coefficient in the simulation is smaller than in HATS for neutral conditions, but experiment and simulation agree very well in unstable conditions. During the evening transition in moderately stable conditions, the scale-dependent coefficient converges to $c_{s} \sim 0.08$, while the scale-invariant coefficient approaches $c_{s} \sim 0.05$ for $z / \Delta_{h}>2.5$. Field experiment and simulation results agree well qualitatively, but the scaledependent coefficients from LES are smaller than the HATS measurements for the moderately stable conditions. In the most stable conditions in the simulation (Figure 9d, $\Delta_{h} / L \sim 4$ ), LES predictions of $c_{s}^{(\Delta)}$ match the a priori results from HATS when the scale-dependent dynamic model is used.

\section{Conclusions}

[41] High resolution large-eddy simulations of unstable and stable atmospheric boundary layers (ABL) with constant surface heat fluxes were conducted using the Lagrangian scale-dependent dynamic SGS model [Bou-Zeid et al., 2005] and the Lagrangian scale-invariant dynamic SGS model [Meneveau et al., 1996]. In unstable conditions, the vertical profiles of mean quantities and fluxes are predicted equally well by both approaches. In stable conditions, there are significant differences in the profiles. The scale-invariant dynamic procedure is underdissipative which leads to larger velocity variances and fluxes in the nocturnal bound- 
ary layer. In addition, a faster growth of the nocturnal boundary layer is observed for the LES with the scaleinvariant dynamic model.

[42] The advantages of the scale-dependent dynamic procedure become especially evident in the velocity spectra, which follow the expected scalings in the inertial range correctly. The spectra in the scale-invariant dynamic simulation are flat, indicating an unnatural buildup of turbulent kinetic energy at the small scales. Obtaining correct velocity and temperature spectra in a simulation is of paramount practical importance, since the energy distribution of turbulence structures greatly affects all transport processes, including those of nonhomogeneous processes such as evapotranspiration over heterogeneous surfaces.

[43] By analyzing the Smagorinsky coefficients obtained during the simulations of a diurnal cycle, we conclude that the Lagrangian dynamic SGS models in LES of ABL flow of varying stability are able to predict trends of the Smagorinsky coefficient $c_{S}^{(\Delta)}$ that agree well with the coefficient measured a priori in the HATS experiment (KMP03, KPM04). $c_{s}^{(\Delta)}$ decreases both in the near-wall region and in stable conditions. The scale invariant dynamic procedure underpredicts the field experimental value of $c_{S}^{(\Delta, \mathrm{emp})}$, but closely matches the scale-invariant coefficients obtained in the field study $c_{s}^{(\Delta, \text { dyn })}$. The Smagorinsky coefficient predicted from the scale-dependent dynamic model is similar to $c_{s}^{(\Delta, \mathrm{emp})}$. However, for neutral and moderately stable conditions $c_{s}^{(\Delta)}$ is larger and increases faster with $z / \Delta_{h}$ in the field measurements than in LES.

[44] The scale-dependent dynamic procedure is successful in automatically reducing $c_{s}^{(\Delta)}$ in stable conditions, such as in the stable region above the inversion layer, and in the nocturnal boundary layer. Moreover, the agreement between LES and field experimental study supports the applicability of a priori studies to gain insights into development and testing of SGS parameterizations for LES. Finally, the detailed analysis of the diurnal cycle simulation of the ABL of Kumar et al. [2006] provides further illustration of the strengths of the dynamic model in LES to study complex time-dependent problems in hydrology and landatmosphere interaction.

[45] Acknowledgments. This study was funded by the National Science Foundation under grants NSF-ATM 01300766 and NSF-WCR 0233646. Computations were made possible with the supercomputing grant from NCAR.

\section{References}

Albertson, J. D., and M. B. Parlange (1999a), Surface length-scales and shear stress: Implications for land-atmosphere interaction over complex terrain, Water Resour. Res., 35, 2121-2132.

Albertson, J. D., and M. B. Parlange (1999b), Natural integration of scalar fluxes from complex terrain, Adv. Water Resour., 23, 239-252.

Bastiaans, R. J. M., C. C. M. Rindt, and A. A. van Steenhoven (1998), Experimental analysis of a confined transitional plume with respect to subgrid-scale modelling, J. Heat Mass Trans., 41, 3989-4007.

Beare, R., et al. (2006), An intercomparison of large-eddy simulations of the stable boundary layer, Boundary Layer Meteorol., in press.

Bou-Zeid, E., C. Meneveau, and M. B. Parlange (2004), Large-eddy simulation of neutral atmospheric boundary layer flow over heterogeneous surfaces: Blending height and effective surface roughness, Water Resour. Res., 40, W02505, doi:10.1029/2003WR002475.

Bou-Zeid, E., C. Meneveau, and M. B. Parlange (2005), A scale-dependent Lagrangian dynamic model for large eddy simulation of complex turbulent flows, Phys. Fluids, 17, 025105.

Brutsaert, W. (2005), Hydrology: An Introduction, Cambridge Univ. Press, New York.
Canuto, V. M., and Y. Cheng (1997), Determination of the SmagorinskyLilly constant $c_{s}$, Phys. Fluids, 9, 1368-1378.

Clark, R. A., J. H. Ferziger, and W. C. Reynolds (1979), Evaluation of subgrid models using an accurately simulated turbulent flow, J. Fluid Mech., 91, 1-16.

Deardorff, J. W. (1974), Three-dimensional numerical study of the height and mean structure of a heated planetary boundary layer, Boundary Layer Meteorol., 7, 81-106.

Deardorff, J. W. (1980), Stratocumulus-capped mixed layers derived from a three dimensional model, Boundary Layer Meteorol., 18, 495-527.

Dyer, A. J. (1974), A review of flux-profile relationships, Boundary Layer Meteorol., 7, 363-374.

Germano, M. (1992), Turbulence: The filtering approach, J. Fluid Mech., $238,325-336$.

Germano, M., U. Piomelli, P. Moin, and W. H. Cabot (1991), A dynamic subgrid-scale eddy viscosity model, Phys. Fluids A, 3, 1760-1765.

Ghosal, S., T. S. Lund, P. Moin, and K. Akselvoll (1995), A dynamic localization model for large eddy simulation of turbulent flow, J. Fluid Mech., 286, 229-255.

Higgins, C. W., M. B. Parlange, and C. Meneveau (2003), Alignment trends of velocity gradients and subgrid-scale fluxes in the turbulent atmospheric boundary layer, Boundary Layer Meteorol., 109, 59-83.

Hogstrom, U. (1987), Non-dimensional wind and temperature profiles in the atmospheric surface layer: A re-evaluation, Boundary Layer Meteorol., 42, 55-78.

Horst, T. W., J. Kleissl, D. H. Lenschow, C. Meneveau, C.-H. Moeng, M. B. Parlange, P. P. Sullivan, and J. C. Weil (2003), Field observations to obtain spatially-filtered turbulence fields from transverse arrays of sonic anemometers in the atmospheric surface layer, J. Atmos. Sci., 61, 15661581.

Hunt, J. C. R., D. D. Stretch, and R. E. Britter (1988), Length scales in stably stratified turbulent flows and their use in turbulence models, in Stably Stratified Flows and Dense Gas Dispersion, edited by J. S. Puttock, pp. 285-322, Clarendon, Oxford, U. K.

Kang, H. S., and C. Meneveau (2002), Universality of large eddy simulation model parameters across a turbulent wake behind a heated cylinder, J. Turbulence, 3, pap. 32, doi:10.1088/1468-5248/3/1/032.

Kleissl, J., C. Meneveau, and M. B. Parlange (2003), On the magnitude and variability of subgrid-scale eddy-diffusion coefficients in the atmospheric surface layer, J. Atmos. Sci., 60, 2372-2388.

Kleissl, J., M. B. Parlange, and C. Meneveau (2004), Field experimental study of dynamic Smagorinsky models in the atmospheric surface layer, J. Atmos. Sci., 61, 2296-2307.

Kosović, B., and J. A. Curry (2000), A large eddy simulation study of a quasi-steady, stably stratified atmospheric boundary layer, J. Atmos. Sci., $57,1057-1068$.

Kumar, V., J. Kleissl, C. Meneveau, and M. B. Parlange (2006), Large-eddy simulation of a diurnal cycle in the turbulent atmospheric boundary layer: Atmospheric stability and scaling issues, Water Resour. Res., 42, W06D09, doi:10.1029/2005WR004651.

Lilly, D. K. (1967), The representation of small-scale turbulence in numerical simulation experiments, in Proceedings of IBM Scientific Computing Symposium on Environmental Sciences, Yorktown Heights, NY, pp. 195210, IBM Data Process. Div., White Plains, N. Y.

Lilly, D. K. (1992), A proposed modification of the Germano subgrid scale closure method, Phys. Fluids A, 4, 633-635.

Liu, S., C. Meneveau, and J. Katz (1994), On the properties of similarity subgrid-scale models as deduced from measurements in a turbulent jet, J. Fluid Mech., 275, 83-119.

Mason, P. J. (1994), Large-eddy simulation: A critical review of the technique, Quart. J. Roy. Meteor. Soc., 120, 1-26.

McMillan, O. J., and J. H. Ferziger (1979), Direct testing of subgrid-scale models, AIAA J., 17, 1340-1346.

Meneveau, C. (1994), Statistics of turbulence subgrid-scale stresses: Necessary conditions and experimental tests, Phys. Fluids A, 6, 815-833.

Meneveau, C. (1996), Transition between viscous and inertial-range scaling of turbulence structure functions, Phys. Rev. E, 54, 3657-3663.

Meneveau, C., and J. Katz (2000), Scale-invariance and turbulence models for large-eddy-simulation, Annu. Rev. Fluid Mech., 32, 1-32.

Meneveau, C., T. Lund, and W. Cabot (1996), A Lagrangian dynamic subgrid-scale model of turbulence, J. Fluid Mech., 319, 353-385.

Moeng, C.-H., and P. Sullivan (1994), A comparison of shear-driven and buoyancy-driven planetary boundary-layer flows, J. Atmos. Sci., 51, 999-1022.

Nieuwstadt, F. T. M., P. J. Mason, C.-H. Moeng, and U. Schumann (1991), Large-eddy simulation of the convective boundary layer: A comparison of four computer codes, Turbulent Shear Flows, 8, 343-367. 
Orszag, S. (1970), Transform method for calculation of vector coupled sums: Application to the spectral form of the vorticity equation, J. Atmos. Sci., 27, 890-895.

Parlange, M. B., and W. Brutsaert (1989), Regional roughness of the Landes forest and surface shear stress under neutral conditions, Boundary Layer Meteorol., 48, 69-81.

Piomelli, U. (1999), Large-eddy simulation: Achievements and challenges, Prog. Aerospace Sci., 35, 335-362.

Piomelli, U., and E. Balaras (2002), Wall-layer models for large-eddy simulation, Annu. Rev. Fluid Mech., 34, 349-374.

Pope, S. B. (2000), Turbulent Flows, Cambridge Univ. Press, New York.

Porté-Agel, F. (2004), A scale-dependent dynamic model for scalar transport in large-eddy simulations of the atmospheric boundary layer, Boundary Layer Meteorol., 112, 81-105.

Porté-Agel, F., C. Meneveau, and M. B. Parlange (2000), A scale-dependent dynamic model for large-eddy simulation: Application to a neutral atmospheric boundary layer, J. Fluid Mech., 415, 261-284.

Redelsperger, J., F. Mahe, and P. Carlotti (2001), A simple and general subgrid model suitable both for surface layer and free stream turbulence, Boundary Layer Meteorol., 101, 375-408.

Scotti, A., C. Meneveau, and D. K. Lilly (1993), Generalized Smagorinsky model for anisotropic grids, Phys. Fluids A, 5, 2306-2308.

Smagorinsky, J. (1963), General circulation experiments with the primitive equations. I. The basic experiment, Mon. Weather Rev., 91, 99164.
Stoll, R., and F. Porté-Agel (2006), Dynamic subgrid-scale models for momentum and scalar fluxes in large-eddy simulations of neutrally stratified atmospheric boundary layers over heterogeneous terrain, Water Resour. Res., 42, W01409, doi:10.1029/2005WR003989.

Stull, R. B. (1997), An Introduction to Boundary Layer Meteorology, Springer, New York.

Sullivan, P. P., T. W. Horst, D. H. Lenschow, C.-H. Moeng, and J. C. Weil (2003), Structure of subfilter-scale fluxes in the atmospheric surface layer with application to large eddy simulation modeling, J. Fluid Mech., 482, $101-139$.

J. Kleissl, Department of Earth and Environmental Sciences, New Mexico Institute of Mining and Technology, 801 Leroy Place, Socorro, NM 87801, USA. (jan@kleissl.com)

V. Kumar, Department of Geography and Environmental Engineering, Johns Hopkins University, Baltimore, MD 21218, USA. (vijayant@jhu. edu)

C. Meneveau, Department of Mechanical Engineering, Johns Hopkins University, Baltimore, MD 21218, USA. (meneveau@jhu.edu)

M. B. Parlange, School of Architecture, Civil and Environmental Engineering, Ecole Polytechnique Fédérale de Lausanne, CH-1015 Lausanne, Switzerland. (marc.parlange@epfl.ch) 